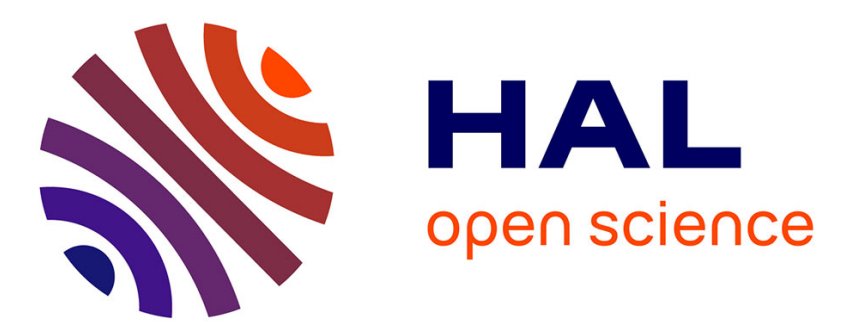

\title{
Dynamic Games for analyzing competition in the Internet and in on-line social networks
}

\author{
Eitan Altman, Atulya Jain, Nahum Shimkin, Corinne Touati
}

\section{To cite this version:}

Eitan Altman, Atulya Jain, Nahum Shimkin, Corinne Touati. Dynamic Games for analyzing competition in the Internet and in on-line social networks. NETGCOOP 2016 - International conference on NEtwork Games. Optimization and Control, Nov 2016, Avignon, France. hal-01378901

\section{HAL Id: hal-01378901 \\ https://hal.inria.fr/hal-01378901}

Submitted on 10 Oct 2016

HAL is a multi-disciplinary open access archive for the deposit and dissemination of scientific research documents, whether they are published or not. The documents may come from teaching and research institutions in France or abroad, or from public or private research centers.
L'archive ouverte pluridisciplinaire HAL, est destinée au dépôt et à la diffusion de documents scientifiques de niveau recherche, publiés ou non, émanant des établissements d'enseignement et de recherche français ou étrangers, des laboratoires publics ou privés. 


\title{
Dynamic Games for analyzing competition in the Internet and in on-line social networks
}

\author{
Eitan Altman ${ }^{1}$, Atulya Jain ${ }^{2}$, Nahum Shimkin ${ }^{3}$, and Corinne Touati ${ }^{4}$
}

\begin{abstract}
The global Internet has enabled a massive access of internauts to content. At the same time it allowed individuals to use the Internet in order to distribute content. This introduced new types of competition between content over popularity, visibility, influence, reputation and user attention. The rules of these competitions are new with respect to those of traditional media, and they are determined by the way resources are allocated through network protocols (such as page rank in search engines and recommendation systems that are widely spread in social networks). In this paper we first present in the introduction an overview of some central competition issues both in the Internet as well as in other types of networks. We then describe the model of when to send content in order to maximize the exposure of the content. In the two last sections we finally describe research on two bio-inspired tools that have bben used to study various competition aspects.
\end{abstract}

\section{Introduction}

Many games that arise in the Internet are not specific to this network. For example the competition for visibility due to limited display space (the computer screen) already appeared under the term shelf-space allocation, see e.g. [27]). Competitive routing in large networks have been studied by road traffic engineers see e.g. [30] which summarizes around one thousand reference. Yet in both examples there are some features that are particular to the Internet. Indeed, (i) advertisement in the internet brings many new features such as targeted advertisement: the social network owner has a huge amount of data on the preferences of his customers and he can thus send the advertisement to those who are more likely to be interested by it. (ii) competitive routing in the Internet is different than rout selection in road traffic since in the former, routing decisions are taken by Internet Service Providers (ISPs) whereas in the latter, each driver takes his own routing decision. Moreover, while ISPs can split their traffic among various paths, a driver takes non-splitable discrete (path selection) decisions. In this survey we focus on those applications of dynamic

\footnotetext{
1 Université Côte d'Azur, INRIA, France, and LINCS, Paris, France e-mail: eitan.altman@inria.fr .2 Université Côte d'Azur, INRIA, France and LIA/CERI, University of Avignon, France e-mail: j.atulya@iitg.ernet.in . ${ }^{3} \mathrm{EE}$ Department, Technion, Israel e-mail: shimkin@ee.technion.ac.il.4 INRIA, Grenoble, France e-mail: Corinne.Touati@inria.fr
} 
games in which features of the internet and of social networks play an important role.

The Internet has introduced new types of competition between content over popularity, visibility, influence, reputation and user attention. Cardon writes in [12] "Whereas journalists filter the information based on human judgement before publishing it, research engines (as well as google news) filter a-posteriori information already published based on human judgement of all the internauts who publish in the WEB. In the numerical world, this is called collective intelligence or crowd wisdom". This is done through algorithms such as page rank which assign authority to a given information in a recursive way, according to the authority assigned to those who reference it. This way of classifying authority of information (of sites, blogs, news, videos, scientific publications) has triggered a whole business of Search Engine Optimization in which companies offer clients to increase their page rank by creating many new hyperlinks to reference their sites. One can view the page rank as a measure of popularity. When searching for information through keywords, google proposes a list of items displayed according to the decreasing order of their page rank. Thus information published on the WEB competes over visibility through a competition over popularity and more popular information is more accessible.

Cardon distinguishes between search engine classification of authority and that of social networks. In twitter, facebook and youtube the source of authority is not in who cites a content but rather in the number of "views" it has, number of "likes", of comments and of "shares" ("retweets" in twitter). Recommendation systems play a crucial role in (i) the visibility of posts in social networks (ii) creating communities by proposing to establish direct links to other members of the network (friendship link in facebook, subscriber link in youtube and follower link in twitter), (iii) building communities by suggesting users to subscribes to groups or to like fan pages. In youtube the recommendations are given in the form of a list of videos that are recommended for watching. The recommendation algorithms could thus have an impact on the composition of the Internet communities and on their evolution. In particular it could determine whether the communities are close or whether there is diversity and dialogue between various tendencies. In [11, p. 92] the author says that Internet algorithms that structure political communities over the WEB have a tendency to create closed groups among people with similar ideas which does not promote diversity.

Internet algorithms can also influence on whether there emerge many small communities or a smaller number of larger ones. In [11, p. 92], the author mentions that many observers are worried about the possibility of having political discussions taking place in many small communities; he calls it a "balkanization" phenomenon. This is just one of the many network formation games that can be observed in the Internet. 


\section{Timing game in social networks: when to post content}

We present below an overview of a game of timing between a random number of content creators, who compete for position and exposure time over the timeline of a social network. The full detailed analysis and related work can be found in [32].

Model Description. Users (or players) can post their items during a given time interval $[0, T]$. The timeline consists of $K \geq 1$ positions, where position 1 is the most effective, and last position $K$ the least effective. The position of the posted items on the timeline is dynamically determined according to their order of arrival: a newly arrived item is placed at the top position, while existing items are pushed one position lower (from 1 to 2, 2 to 3 etc.). The item at position $K$ (if any) is ejected and dropped the timeline.

For $t \in[0, T]$ and $k=1, \ldots, K$, let $u_{k}(t)$ denote the expected utility rate (per unit time) for a displayed item at time $t$ in position $k$. The total expected utility over the entire life cycle of an item is therefore

$$
U\left(\mathscr{T}_{1}, \ldots, \mathscr{T}_{K}\right)=\sum_{k=1}^{K} \int_{t \in \mathscr{T}_{k}} u_{k}(t) d t
$$

where $\mathscr{T}_{k}$ is the time interval on which the Item was displayed at position $k$. We assume that the functions $u_{k}(t)$ are decomposed as

$$
u_{k}(t)=r_{k} u(t), \quad t \in[0, T], k=1, \ldots, K,
$$

where

- The exposure function $u(t)$, which is common to all positions, captures the temporal dependence of the utility, due to variation in the exposure of the entire timeline.

- The constants $\left(r_{k}, k=1, \ldots, K\right)$ are the relative utility parameters, which capture the relative effectiveness of the different positions in the timeline. That is, the relative utilities are positive, and are decreasing in the timeline position $k$. It will be convenient for our analysis to define $r_{k}=0$ for $k \geq K+1$.

The following assumptions are imposed throughout the paper.

\section{Assumption 2.1}

(i) The exposure function $u:[0, T] \rightarrow \mathbb{R}$ is continuous and strictly positive, namely $u(t)>0$ for $t \in[0, T]$. Let $u_{\min }>0$ and $u_{\max }$ denote the extremal values of $u$.

(ii) The relative utility parameters $\left(r_{k}\right)$ are decreasing in the timeline position. Specifically,

$$
r_{1}>r_{2} \geq r_{3} \cdots \geq r_{K}>0 \text {. }
$$

The game formulation involves several players, who compete for a place in the timeline and wish to to maximize their individual utilities. Each player $i$ chooses the submission time $t_{i}$ of his own item. As mentioned, upon submission, the item is placed in the top position, but goes down in rank as further items are posted. Clearly, 
the utility of each player depends on his or her own choice of submission time $\left(t_{i}\right)$, as well as the submission times of the other player. We therefore consider the problem as a non-cooperative game, and analyze the Nash equilibrium of this game.

To complete the game description, we specify some additional properties.

1. The number of players who participate in a given instance of the game is a random variable, denoted $D_{0}$. We refer to $D_{0}$ as the objective demand.

2. The belief of each participating player regarding the number of other players in the game is another random variable, denoted $D$. We refer to $D$ as the subjective demand. Clearly, if $D_{0}$ is deterministic then $D=D_{0}-1$. The general relation between $D$ and $D_{0}$ is discussed in [32]. Let $p_{D}=\left(p_{D}(n), n \geq 0\right)$ denote the distribution of $D$. We assume that $E(D)<\infty$, and further, to avoid triviality, that $r_{D+1}<r_{1}$ with positive probability ${ }^{1}$.

3. A player cannot observe the submission times of others before choosing his own submission time; in particular, the players do not observe the timeline status before their arrival. (As we shall see, the latter assumption can be relaxed when $D$ follows a Poisson distribution.)

4. The submission time $t_{i}$ of player $i$ can be chosen randomly, according to a probability distribution on $[0, T]$ with cumulative distribution function $F_{i}(t), t \in[0, T]$. The corresponding density function, when it exists, will be denoted by $f_{i}(t)$. We refer to $F_{i}$ as the (mixed) strategy of player $i$.

We shall be interested in the Nash equilibrium point (NEP) of this game. Specifically, we consider the symmetric NEP in which the strategies of all players are identical, namely $F_{i} \equiv F$ all players. The restriction to symmetric strategies, besides its analytical tractability, seems natural in the present scenario where players are essentially anonymous. We proceed to calculate the players' utilities for the symmetric case.

Expected Utility. Consider a certain player $i$ who posts his item at time $t$. Suppose that each of the other $D$ players uses an identical strategy $F$. We proceed to calculate the expected utility $U(t ; F)$ of the player in that case.

Suppose first that $F$ has no point mass at $t$, so that with probability 1 there are no simultaneous arrivals at $t$. Let $N_{(t, s]}$ denote the number of arrivals (by other players) during the time interval $(t, s]$, for $t<s \leq T$. Since $i$ arrives at $t$, his position in the timeline at time $s$ will be $k+1$ if $N_{(t, s]}=k$, for $0 \leq k \leq K-1$, and he would have left the timeline if $N_{(t, s]} \geq K$. It follows that

$$
\begin{aligned}
U(t ; F) & =E_{F}\left(\int_{t}^{T} \sum_{k=0}^{K-1} r_{k+1} \mathbf{1}_{\left\{N_{(t, s]}=k\right\}} u(s) d s\right) \\
& =\int_{t}^{T} \sum_{k=0}^{K-1} r_{k+1} \mathbb{P}_{F}\left(N_{(t, s]}=k\right) u(s) d s .
\end{aligned}
$$

\footnotetext{
${ }^{1}$ Otherwise, all $D+1$ players can arrive at $t=0$ and remain in positions with maximal relative utility $r_{1}$ all the way up to $T$.
} 
To compute the probability $\mathbb{P}_{F}\left(N_{(t, s]}=k\right)$, recall that the number of participating players other than $i$ is a random variable $D$. The probability that each of these players submits his item on $(s, t]$ is $F(s)-F(t)$. Therefore, conditioned on $D=n, N_{(t, s]}$ follows a $\operatorname{Binomial}$ distribution $\operatorname{Bin}(n, p)$ with success probability $p=F(s)-F(t)$. Denoting

$$
B_{k, n}(p)=\left(\begin{array}{l}
n \\
k
\end{array}\right) p^{k}(1-p)^{n-k}, \quad 0 \leq k \leq n
$$

(and setting $B_{k, n} \equiv 0$ for $k>n$ ), we obtain

$$
\begin{aligned}
\mathbb{P}_{F}\left(N_{(t, s]}=k\right) & =\sum_{n \geq k} p_{D}(n) \mathbb{P}_{F}\left(N_{(t, s]}=k \mid D=n\right) \\
& =\sum_{n \geq k} p_{D}(n) B_{k, n}(F(s)-F(t)) .
\end{aligned}
$$

Substituting (3) in (1) gives

$$
U(t ; F)=\int_{t}^{T} \sum_{k=0}^{K-1} r_{k+1} \sum_{n \geq k} p_{D}(n) B_{k, n}(F(s)-F(t)) u(s) d s .
$$

We mention some special cases of this expression.

Single Position timeline: When $B=1$, noting that $B_{0, n}(p)=(1-p)^{n}$ we obtain

$$
U(t ; F)=r_{1} \int_{t}^{T} \sum_{n \geq 0} p_{D}(n)(1-F(s)+F(t))^{n} u(s) d s .
$$

Deterministic Demand: Suppose $D$ is a deterministic positive integer (which corresponds to a game with $D_{0}=D+1$ players). In that case

$$
\mathbb{P}_{F}\left(N_{(t, s]}=k\right)=B_{k, D}(F(s)-F(t)),
$$

and

$$
U(t ; F)=\int_{t}^{T} \sum_{k=0}^{K-1} r_{k+1} B_{k, D}(F(s)-F(t)) u(s) d s .
$$

Poisson Demand: Suppose $D \sim \operatorname{Pois}(\Lambda)$, a Poisson random variable (RV) with parameter $\Lambda>0$, namely $p_{D}(n)=\Lambda^{n} e^{-\Lambda} / n$ ! for $n \geq 0$. Since a Bernoulli dilution of a Poisson RV remains Poisson, it follows that $N_{(t, s]}$ is a Poisson RV with parameter $\Lambda(F(s)-F(t))$, and

$$
\mathbb{P}_{F}\left(N_{(t, s]}=k\right)=\frac{1}{k !} \Lambda^{k}(F(s)-F(t))^{k} e^{-\Lambda(F(s)-F(t))} .
$$

This expression can be directly substituted in Equation (1).

Simultaneous arrivals: If $F$ has a point mass at $t$, then there is a positive probability of simultaneous arrivals of several players at that time. In that case we assume that their order of arrival (and subsequent positioning on the timeline) is determined uniformly at random. The utility $U(t ; F)$ needs to be modified accordingly. We need 
not bother here with writing the straightforward but cumbersome expression, as we argue below that in equilibrium $F$ does not possess point masses.

Nash Equilibrium. A symmetric Nash equilibrium point (NEP) is represented by a strategy $F$, which is a probability distribution on $[0, T]$, such that $F$ is a best response for each player when all others use the same strategy $F$. More formally, for any pair of strategies $G$ and $F$, let

$$
\bar{U}(G ; F)=E_{t \sim G}(U(t ; F))=\int_{t} U(t ; F) d G(t)
$$

denote the expected utility of a player for using strategy $G$ when all others follow $F$. Then $F$ represents a symmetric NEP if

$$
F \in \underset{G}{\operatorname{argmax}} \bar{U}(G ; F),
$$

where the maximum is taken over all probability distributions on $[0, T]$. We shall refer to a symmetric equilibrium strategy $F$ as an equilibrium profile.

An equivalent definition of the symmetric NEP, that is more useful for the analysis, requires $U(t ; F)$ to be minimized on a set of times $t$ of $F$-probability 1 . That is: There exists a constant $u^{*}$ and a set $A \subset[0, T]$, such that $\int_{A} d F(t)=1$, and

$$
\begin{aligned}
& U(t ; F)=u^{*} \text { for } t \in A, \\
& U(t ; F) \leq u^{*} \text { for } t \notin A .
\end{aligned}
$$

The equivalence of the two definitions is readily verified. We refer to the constant $u^{*}=u_{F}^{*}$ as the equilibrium utility corresponding to an equilibrium profile $F$.

Equilibrium Analysis. For an arrival profile $F$ and $t \in[0, T]$, denote

$$
g(t, F)=\sum_{k=0}^{K-1}\left(r_{k+1}-r_{k+2}\right) \sum_{n \geq k}(n+1) p_{D}(n+1) \int_{t}^{T} B_{k, n}(F(s)-F(t)) u(s) d s .
$$

Let $F^{\prime}(t)$ denote the time derivative of $F$ at $t$. Recall that the support of a probability measure $\eta$ is the smallest closed set of $\eta$-probability 1 . For brevity, we denote by $\operatorname{supp}(F)$ the support of the probability measure $\eta_{F}$ induced by a distribution function $F$. Finally, recall that $U(t ; F)$ is the expected utility which is specified in (4).

Theorem 1 (Existence and Characterization). An equilibrium profile $F=(F(t), t \in$ $[0, T])$ exists. Any equilibrium profile satisfies the following properties.

(i) $F$ is a continuous function, and there exists a number $L \in(0, T)$ such that $\operatorname{supp}(F)=$ $[0, L]$. Specifically, $F(0)=0, F(L)=1$, and $F(t)$ is strictly increasing in $t \in[0, L]$.

(ii) Consequently, a continuous probability distribution function $F$ on $[0, T]$ is an equilibrium profile if, and only if, there exists a number $L \in(0, T)$ such that $F(0)=0$, $F(L)=1$, and $U(t ; F)=u_{L}$ for $t \in[0, L]$ and some constant $u_{L}>0$.

(iii)Equivalently, a continuous probability distribution function $F$ on $[0, T]$ is an equilibrium profile if, and only if, there exists a number $L \in(0, T)$ such that: $F(0)=0$, $F(L)=1$, and the derivative $F^{\prime}(t)$ exists for $t \in(0, L)$ and satisfies the equality 


$$
F^{\prime}(t)=\frac{r_{1} u(t)}{g(t, F)}, \quad t \in(0, L),
$$

where $g(t, F)$ is defined in (7).

(iv) For an equilibrium profile $F$ with support $[0, L]$, the equilibrium utility $u_{F}^{*}$ is given by $u_{F}^{*}=r_{1} \int_{L}^{T} u(s) d s$.

Assumption 2.2 The relative utility parameters satisfy the following convexity condition: $r_{k} \leq \frac{1}{2}\left(r_{k-1}+r_{k+1}\right), \quad k=2, \ldots, K$ (recall that $r_{K+1}=0$ by definition).

Theorem 2 (Uniqueness). Suppose Assumption 2.2 holds, then the equilibrium profile $F$ is unique.

\section{Stochastic evolutionary games}

We describe in this section and in the next one some novel bio-inspired tools in dynamic games that have been developed and used recently in networking applications and in which we expect there to be further research both in the fundamentals as well as in the applications.

Evolutionary games are considered as dynamic since they describe through differential equations the evolution of the strategies in some repeated games when the system is away from equilibrium. In many applications that we encountered in networking there is a need, however, for a fully dynamic system in the sense of not only the action evolution but also of a state evolution, a state that may be attached to each agent. An example is power control in cellular networks in which the chosen power may depend on the channel state (which is a function of the weather, the distance to the base station and other factors). In some cases this state may have controlled transitions, so that the choice of action (when interacting with other players) impacts not only the immediate fitness of the agents but also the agents' state. For example, the choice of transmission power of a cellular network may be depend on the state of its battery, and when the battery is depleted, a saving mode code be activated in which power is used much more carefully. We next describe two frameworks that model evolution in presence of controlled states of the agents.

In $[3,4]$ one such game model called Markov Decision Evolutionary Game (MDEG) is introduced where indeed each player has an internal state. The fitness received in a local interaction with another player depends then not only on the actions chosen but also on the internal states of the players. Moreover, the internal states of individuals that interact change with probabilities that depend on both the actions and the internal states of the individuals. Finally, an individual's objective is not to maximize its fitness (as is the case with standard EVGs) but rather to maximize its sum of expected fitness during its lifetime, or its time-average fitness. We have been able so far to use this model in several problems in wireless communications and computed the equilibrium.

A second approach for adding states and randomness in their controlled evolution is known as Sequential Anonymous Games (SAG). This class of games introduced 
in [19] has a structure similar to MDEG. The difference is that in MDEGs, strategies interact through pairwise interactions between players, whereas in SAGs, the interactions involve a cumulative effect of an infinite class of players. As in MDEGs, each player has its own Markov chain whose transition probabilities depend on the state and action of that player as well as the global state and the policy used by other players. The discounted cost has been studied already in [19] for characterizing the equilibrium, whereas the expected time average fitness has only been studied recently [36]. This reference as well as [5] include some networking applications.

\section{Epidemic games for cyber-security and content diffusion}

Computer viruses have been reported to cause damage of 17 billion US\$ on 2000. Already in 1998, the relation between computer viruses and epidemiology are suggested [28]. Since then, viruses and tools to fight them have become more sophisticated. Today cyber security is not only defensive and are used as warfare, see [20].

In the biology literature, there has been almost no research using game models in epidemics. We found no references to games in epidemics before 2000. Some isolated research on the topic has appeared in recent years $[6,7,9,8]$. These papers use simple game theoretical tools to model some decisions related to fighting against viruses (namely vaccination decisions and decisions concerning observations of epidemics). [31] is a recent paper using a differential population game for solving an epidemic game arising in the biological context.

Optimal control theory has been used within the classical epidemic models so as to (i) identify the worst possible computer-viruses attacks in a given network (ii) to fight viruses whose behavior is described by classical epidemic models. In [21], the authors combine (i) and (ii) within a single differential game formulation and obtain the structure of the saddle-point policies in the meanfield regime. These results are also novel with respect to epidemiology (and not only computer viruses).

For game theory applied to other aspects of security issues in networks we refer to the survey [26].

A big boost to games in epidemics was given thanks to work by the group of P. Van Mieghem [24, 25] which provides both bounds on the dynamics of SIS epidemic models as well as meanfield approximations on their metastable regime. It was mainly followed in the physics community who are interested in phase transitions and in meanfield dynamics. The complexity of the solution is significantly reduced in the meanfield regime (in which the number of agents grows to infinity) due to the property that the infection probability of any two nodes becomes independent in that regime. The accuracy of the mean field approach is studied in [25]. While in the physics community, this type of approach in studying epidemics is relatively recent, the use of the meanfield approximation is well known in other communities. Indeed, already [22, 33, 23] establish conditions for convergence to a meanfield regime, and Mandelbaum and Pats apply it to epidemics in [23, Sec. 10]. 
Game models (static ones) based on the above SIS epidemic theory appear already in 2009 [29] and later at [17]. In these game each node is a player. Recently, Nash and Stackelberg epidemic adversarial games have been introduced in [37] where one player controls the epidemic rate and the other controls the curing rate. Both in static as well as in dynamic games based on meanfield limits, one has to be aware that the fact that a meanfield approach is a good approximation for a noncontrolled system or for a fixed control does not imply that the equilibrium of a meanfield game is a good approximation for a game with a large finite population of players. Conditions for the latter to hold can be found in $[18,15,16]$. A Counter example is presented in [14].

The majority of recent works on epidemic games is based on formulating static games based on steady state (or metastable) performance measures of fixed stationary policies used in Markov dynamic setting, see e.g. [2] that considers games in online dating platforms, or [35] that considers network formation games in which the utilities includes a term representing the metastable infection probability. Some examples of fully dynamic cybersecurity epidemic games are [21] (already mentioned) as well as [10].

Epidemics and their control have also been used to model diffusion of content in social networks. Already the evolutionary biologist Richard Dawkins shows that the evolution of phenomena such as fashion, musical melodies and other cultural phenomena (for which he coined the term "meme") follows similar rules as the Darwinian evolution of species in nature, see [13] p 192. Dawkins later adapted this characterization to Internet Memes as well [34], which includes hits and videos that compete for popularity and visibility over the Internet. Epidemic models can thus be introduced to games in which a virus represents some content.

\section{Acknowledgement}

The work of the second author was partly supported by IFCAM (Indo-French Centre for Applied Math).

\section{References}

1. Eitan Altman, "A stochastic game approach for competition over popularity in social networks", Dynamic Games and Applications 3, 2 (2013) 313-323.

2. Eitan Altman, Francesco De Pellegrini, Huijuan Wang, "Activation Games in Online Dating Platforms", IEEE ICCW, Jun 2015, London, United Kingdom. pp.1593-1599

3. E. Altman and Y Hayel, "Stochastic Evolutionary Games", Proc. of the 13th Symposium on Dynamic Games and Applications, Wroclaw, Poland, 30th June-3rd July, 2008.

4. E. Altman, Y. Hayel, H. Tembine and R. El-Azouzi, "Markov Decision Evolutionary Games with Expected Average Fitness", Evolutionary Ecology Research, 11 (4):677-689, 2009

5. Eitan Altman, Piotr Wiecek, "Applications of Stationary Anonymous Sequential Games to Multiple Access Control in Wireless Communications", International Workshop on Wireless 
Networks: Communication, Cooperation and Competition, May 2014, Hammamet, Tunisia. pp.575-578, 2014.

6. J. Aspnes, K. L. Chang, and A. Yampolskiy. "Inoculation strategies for victims of viruses and the sum-of-squares partition problem”. J. Comput. Syst. Sci., 72(6):1077=1093, 2006.

7. J Aspnes, N Rustagi, and Saia. "Worm versus alert: Who wins in a battle for control of a largescale network?", volume 4878 of Lecture Notes in Computer Science, Springer, 443-456. Dec 2007.

8. C T Bauch. "Imitation dynamics predict vaccinating behavior". Proc. of The Royal Society, 2005.

9. C. T. Bauch and D. J. D. Earn. Vaccination and the theory of games. Proceedings of the National Academy of Science, 101:13391-13394, September 2004.

10. Alain Bensoussan, Murat Kantarcioglu and SingRu(Celine) Hoe, "A Game-Theoretical Approach for Finding Optimal Strategies in a Botnet Defense Model", T. Alpcan, L. Buttyan, and J. Baras (Eds.): GameSec 2010, LNCS 6442, pp. 135-148, 2010.

11. Dominque Cardon, The digital democracy (in French), Seuil, 2010

12. Dominique Cardon, What do Algorithms Dream of (in French), Seuil 2015

13. R. Dawkins, The Selfish Gene, Oxford University Press, 1989.

14. Josu Doncel, Nicolas Gast, Bruno Gaujal, "Are mean-field games the limits of finite stochastic games?" The 18th Workshop on MAthematical performance Modeling and Analysis, Jun 2016, Nice, France. Performance evaluation review (PER), 2016. Available in HAL repository, https://www.archives-ouvertes.fr/hal-01321020/

15. Josu Doncel, Nicolas Gast, Bruno Gaujal, "Mean-Field Games with Explicit Interactions", 2016, Available in HAL repository at https://hal.inria.fr/hal-01277098.

16. Josu Doncel, Nicolas Gast, Bruno Gaujal, "A mean-field game with explicit interactions for epidemic models", Proceedings of the 11th Atelier of Performance Evaluation, Toulouse, 11517 March, 2016.

17. Y. Hayel, S. Trajanovski, E. Altman, H. Wang, and P. V. Mieghem, "Complete game-theoretic characterization of sis epidemics protection strategies," in Proc. 53rd IEEE Conference on Decision and Control (CDC), 2014.

18. Hamidou Tembine, Jean-Yves Le Boudec, Rachid El-Azouzi, Eitan Altman, "Mean field asymptotics of Markov decision evolutionary games and teams", GameNets' 2009.

19. B. Jovanovic and R.W. Rosenthal, "Anonymous Sequential Games", J Math Economics 17:7787,1988 .

20. M. B. Kelley, "The Stuxnet attack on Irans nuclear plant was far more dangerous than previously thought," Online: http://www.businessinsider.com/stuxnet-was-far-more-dangerousthanprevious-thought-2013-11, accessed: June, 2014.

21. M.H.R. Khouzani, S. Sarkar and E. Altman, "Saddle-Point Strategies in Malware Attack", IEEE Journal on Selected Areas in Communications, Vol. 30, No. 1, January 2012.

22. T. G. Kurtz. Approximation of population processes, volume 36. SIAM, 1981.

23. Avi Mandelbaum and Gennady Pats, "State-dependent stochastic networks. Part I: Approximations and applications with continuous diffusion limis," The Annals of Applied Probability, 8(2), 569-646, 1998

24. P. Van Mieghem, J. Omic, and R. Kooij, "Virus spread in networks," IEEE/ACM Transactions on Networking, vol. 17, no. 1, pp. 1-14, 2009.

25. Van Mieghem, P. and R. van de Bovenkamp, 2015, "Accuracy criterion for the mean-field approximation in SIS epidemics on networks", Physical Review E, Vol. 91, No. 3.

26. M.H. Manshaei, Q. Zhu, T. Alpcan, T. Basar, and J.-P. Hubaux. "Game theory meets network security and privacy". ACM Computing Survey, 45(3):25:1-25:39, June 2013.

27. Guiomar Martín-Herrän and Sihem Taboubi, "Incentive Strategies for Shelf-Space Allocation in Duopolies", in Dynamic Games: Theory and Applications, A. Haurie and G. Zaccour (edrs), Springer, pp 231-253, 2005.

28. W. Murrey, "The application of epidemiology to computer viruses". Comp. Security 7:139150,1988 
29. J. Omic, A. Orda, and P. V. Mieghem, "Protecting against network infections: A game theoretic perspective," in Proceedings of INFOCOM, 2009, pp. 1485-1493.

30. M. Patriksson, The traffic assignment problem: models and methods, VSP, 1991.

31. Timothy C. Reluga, "Equilibria of an Epidemic Game with Piecewise Linear Social Distancing Cost", Bulletin of Mathematical Biology, October 2013, Volume 75, Issue 10, pp 1961-1984.

32. Eitan Altman, Nahum Shimkin, "The Ordered Timeline Game: Strategic Posting Times Over a Temporally Ordered Shared Medium", Dynamic Games and Applications, Springer Verlag, 2015, pp.1-25.

33. Adam Shwartz and Alan Weiss, Large Deviations for Performance Analysis, Chapman and Hall, 1995.

34. O. Solon, "Richard Dawkins on the internet's hijacking of the word 'meme'". Wired UK. July 9, 2013.

35. Stojan Trajanovski, Fernando Antonio Kuipers, Yezekael Hayel, Eitan Altman, Piet Van Mieghem, "Designing virus-resistant networks: a game-formation approach", CDC, Dec 2015, Osaka, Japan.

36. Piotr Wiecek, Eitan Altman, "Stationary Anonymous Sequential Games with Undiscounted Rewards", Journal of Optimization Theory and Applications, Springer Verlag, 2015, 166 (2), pp.1-25.

37. Z. Xu, A. Khanafer, and T. Basar. Competition over epidemic networks: Nash and Stackelberg games. Proc. 2015 American Control Conference (ACC 2015), Chicago, IL, July 1-3, 2015, pp. 2063-2068. 